This document is the accepted manuscript version of the following article:

Henninen, T. R., Bon, M., Wang, F., Passerone, D., \& Erni, R. (2019). The structure of sub-nm platinum clusters at elevated temperatures. Angewandte Chemie International Edition. https://doi.org/10.1002/anie.201911068

\title{
The Structure of Sub-nm Platinum Clusters at Elevated Temperatures
}

Authors: Trond R. Henninen, ${ }^{\star *}$ Marta Bon, ${ }^{\dagger}$ Feng Wang, ${ }^{\dagger}$ Daniele Passerone, ${ }^{\ddagger}$ Rolf Erni ${ }^{\dagger *}$

Affiliations: $\left(^{\dagger}\right)$ Electron Microscopy Center, $\left(^{\ddagger}\right)$ nanotech@surfaces Laboratory, Empa, Swiss Federal Laboratories for Materials Science and Technology, CH-8600 Dübendorf, Switzerland.

*Correspondence to: trond.henninen@empa.ch, $\underline{\text { rolf.erni@empa.ch }}$

Keywords: Cluster compounds; Platinum; Electron microscopy; Structure elucidation; Nucleation

\section{Abstract}

Little is known about metallic clusters consisting merely of a dozen of atoms or even less, despite of their importance in catalysis and crystal nucleation. Scanning transmission electron microscopy (STEM) provides direct atomic structure information but has inherently suffered from limited time resolution. We employ fast dynamic STEM combined with a spatio-temporal image denoising algorithm to explore the structure and stability of Pt clusters on carbon, which represents a highly relevant catalysis system. At room temperature, dynamic amorphous $2 \mathrm{D}$ structures are found, while above $\sim 300^{\circ} \mathrm{C}$, the clusters transform into a crystalline state. Our experimental and theoretical data reveal an unexpected strong trend of the crystalline clusters to exhibit the face-centered cubic, bulk structure of Pt with cuboidal geometries being most prominent.
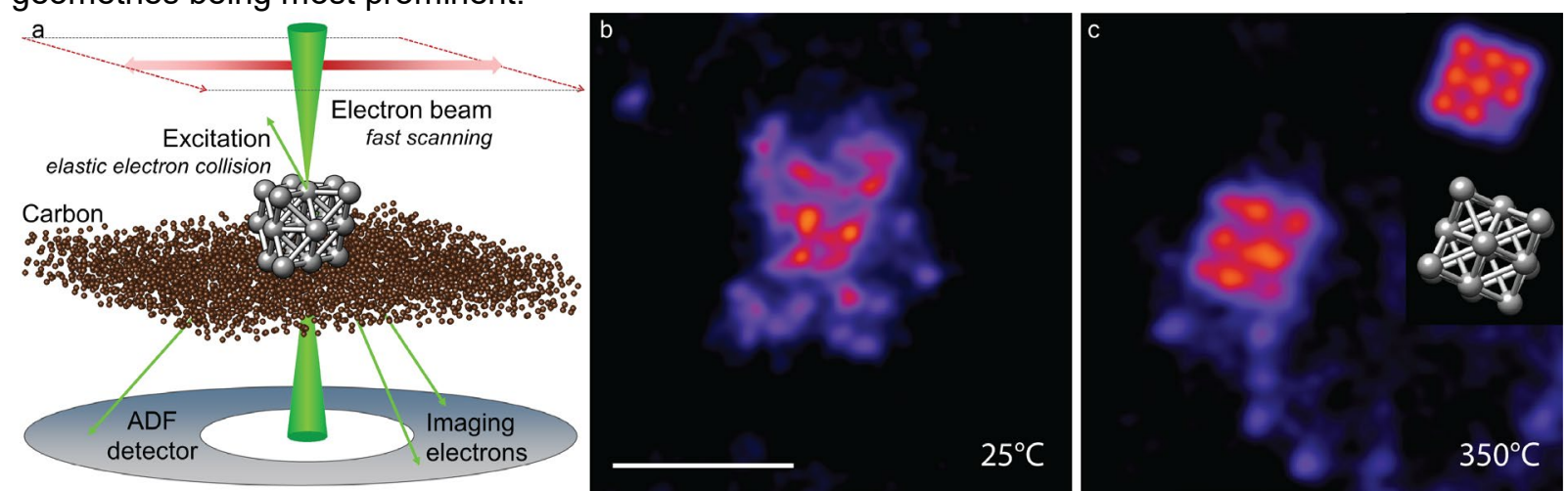

Figure 1: Fast STEM imaging of Pt clusters at variable temperatures. (a) Schematic of the experimental system. (b-c) Single shot, denoised, false coloured STEM images showing (b) a typical amorphous Pt cluster at room temperature (frame 1, Video S1), and (c) a crystalline $\mathrm{Pt}_{17}$ fcc cluster at $350^{\circ} \mathrm{C}$ (frame 22 , Video S2). Inset is its simulated image and atomic model derived from a series of images. $1 \mathrm{~nm}$ scale bar. 
During the initial stages of crystal growth, the building blocks of the future crystalline particle continuously assemble, change into different metastable embryos and disassemble if the free energy does not favor it. The nucleation hurdle is defined by the surface energy of the new phase, and more realistically also by additional factors like shape, symmetry and local composition. The importance of crystallization can be understood in light of its relevance in different industrial areas, such as pharmaceutical, metallurgic, and energy storage sectors. ${ }^{[1]}$ In this respect, understanding nucleation reactions allows an improved control over nanomaterial fabrication, and potentially creating new materials. ${ }^{[2]}$ Nucleation has thus been intensively studied, albeit with little experimental findings when it comes to understanding the very early stages of atom agglomeration, namely pre-nucleation clustering. For $\mathrm{Pt}$, the importance of understanding the structure of its clusters goes beyond crystal nucleation. Nanoparticles and clusters of Pt are used as catalysts in numerous chemical reactions, such as oxidative dehydrogenation of propane,$^{[3]}$ chemical conversion of methane, ${ }^{[4]}$ styrene hydrogenation, ${ }^{[5]}$ electrooxidation of $\mathrm{CO},{ }^{[6]}$ hydrogen evolution reaction, ${ }^{[7]}$ and catalytic conversion of car exhaust. The catalytic activity of small Pt clusters increases with decreasing cluster size due to the large fraction of low-coordinated surface atoms. ${ }^{[4-6,8]}$ Therefore, research has aimed at understanding structure and properties of single site catalysts of sub-nm Pt clusters. ${ }^{[3,5-7]}$

Observations by (scanning) transmission electron microscopy (STEM/TEM) have shown that tiny nanoparticles can exhibit a quasimolten behavior where the crystal frequently transforms between multiple morphologies. ${ }^{[9-12]}$ Yet, these efforts have been limited in spatial resolution and sensitivity to nanometer sized particles. Aberration-corrected annular dark-field STEM (ADF-STEM) has boosted the possibility of interpreting 3D structures of clusters and their dynamics. ${ }^{[13-17]}$ However, such dynamic electron optical imaging is inherently limited by the signal-to-noise ratio (SNR) of the resulting images. After applying spatio-temporal denoising, this strategy has enabled imaging at a speed approaching 10 frames/s (fps). ${ }^{[18,19]}$

To overcome this limitation, we optimized the microscope for fast dynamic STEM (see Methods). With dwell times per pixel of less than $100 \mathrm{~ns}$, only a few tens of electrons pass through the sample per scan 
position and only a fraction of these are scattered to the detector, as illustrated in Fig. 1a. This imaging mode thus results in noisy images (SNR $\sim 0 \mathrm{~dB})$, which were processed using spatio-temporal denoising $^{[18]}$ to extract reliable structural data. Our approach enables fast atomic resolution imaging at an image quality sufficient to interpret the 3D structure of sub-nm Pt clusters, as they continuously rotate and reconstruct. Crystalline structures appear more frequently as the temperature increases, with a preference towards cuboidal-fcc (face-centred cubic) geometries. These experimental findings are supported by density-functional theory (DFT) calculations.

\section{RESULTS AND DISCUSSION}

Initially, we were interested in the structure of the clusters at room temperature (RT). Our observations show that clusters smaller than about $1.5 \mathrm{~nm}$ ( $\sim 50$ atoms), are amorphous with no apparent ordered structure. These clusters are largely 2-dimensional, spreading out similar to a liquid wetting the carbon surface. Fig. 1b depicts a typical amorphous cluster of $36 \pm 1$ atoms, where we see a 2D-like Pt sheet with occasional brighter spots where 2-3 atoms overlap. The clusters behave highly dynamically at RT, continuously undergoing structural rearrangements of uncorrelated atomic motions. During a one minute recording, such amorphous clusters typically disintegrate into smaller fragments and individual atoms (Video S1). This is a direct consequence of the $300 \mathrm{kV}$ electron beam irradiation, which apart from imaging, also challenges the stability of the structures as a single $300 \mathrm{keV}$ electron can transfer up to 4.4 $\mathrm{eV}$ to a Pt atom by elastic collision.

Secondly, we addressed the question of the clusters' temperature dependence, motivated by the fact that catalysts operate at elevated temperatures. Although the electron beam challenges the stability of the clusters, our results clearly reveal that at temperatures approaching $300^{\circ} \mathrm{C}$, the amorphous clusters gradually transform into ordered crystalline structures. An example is seen in Fig. 1c, recorded at $350^{\circ} \mathrm{C}$. Given the fact that this crossover occurs over a wide temperature range, we cannot identify a classical amorphous-to-crystalline phase transition, but we suspect that increasing temperature helps overcoming high free energy barriers towards stable ordered structures. Furthermore, it seems likely that these barriers must vary with the cluster size. Since beam damage reduces crystallinity, which is lost at too high 
dose rates (Video S3), optimization of the dose rate is important to observe these ordered structures. In contrast to the amorphous ones, the crystalline clusters stay intact through extended periods of radiation exposure. After heat treatment at a temperature high enough to reach crystallinity and subsequent cooling down to RT, the clusters become amorphous again, making the crossover reversible (see Fig. S1).

Preliminary experiments with sub-nm Au clusters on a C-film (Fig. S1) show a similar behaviour to Pt on C-film with crystallinity apparent above $200^{\circ} \mathrm{C}$. This suggests that a similar amorphous-to-crystalline crossover can also occur with metal clusters other than Pt. Yet, in order to investigate the possible effect of the substrate, we performed a similar experiment with Pt sputtered on SiN. In this case, the 2D wetting effect at RT was substantially reduced with the clusters being more 3D and structured (see Fig. S2). Furthermore, on the SiN support, a temperature dependence of the crystallinity is not apparent, and the clusters tend to be less stable compared to the experiment on C surface. This, in agreement with the DFT calculations discussed below, underlines the importance of the choice of the substrate in our experiment and underlines the fact that we observe cluster dependencies which are not dominated by the electron irradiation.

Next, we aimed at characterizing the 3D crystalline state of the clusters based on series of images (for details see Methods). At temperatures above $300^{\circ} \mathrm{C}$, the majority of $3 \mathrm{D}$ structures were classified as being fcc structured. While the fcc structure is the stable bulk structure of $\mathrm{Pt}$, this observation contrasts with the majority of literature data which tend to favor icosahedral structures for clusters of similar sizes [20-22]. Notably, the tendency to fcc stabilization is well present even for the smallest crystalline clusters found so far, namely the 6-atom octahedrons. Two such clusters are seen in Fig. S3, where they can be seen from multiple orientations as they rotate while attaching onto larger neighboring clusters. Among the fcc geometries are cuboidal clusters, varying in size and shape, from cubic (Fig. 2a,b) to rectangular cuboids (Fig. 2c,d). Compared to other fcc structures, the cuboidal clusters are less dynamic. While other clusters rotate continuously, the cuboids show a preference for specific orientations towards the carbon surface, and make distinct rotations among these (see Fig. 2). 

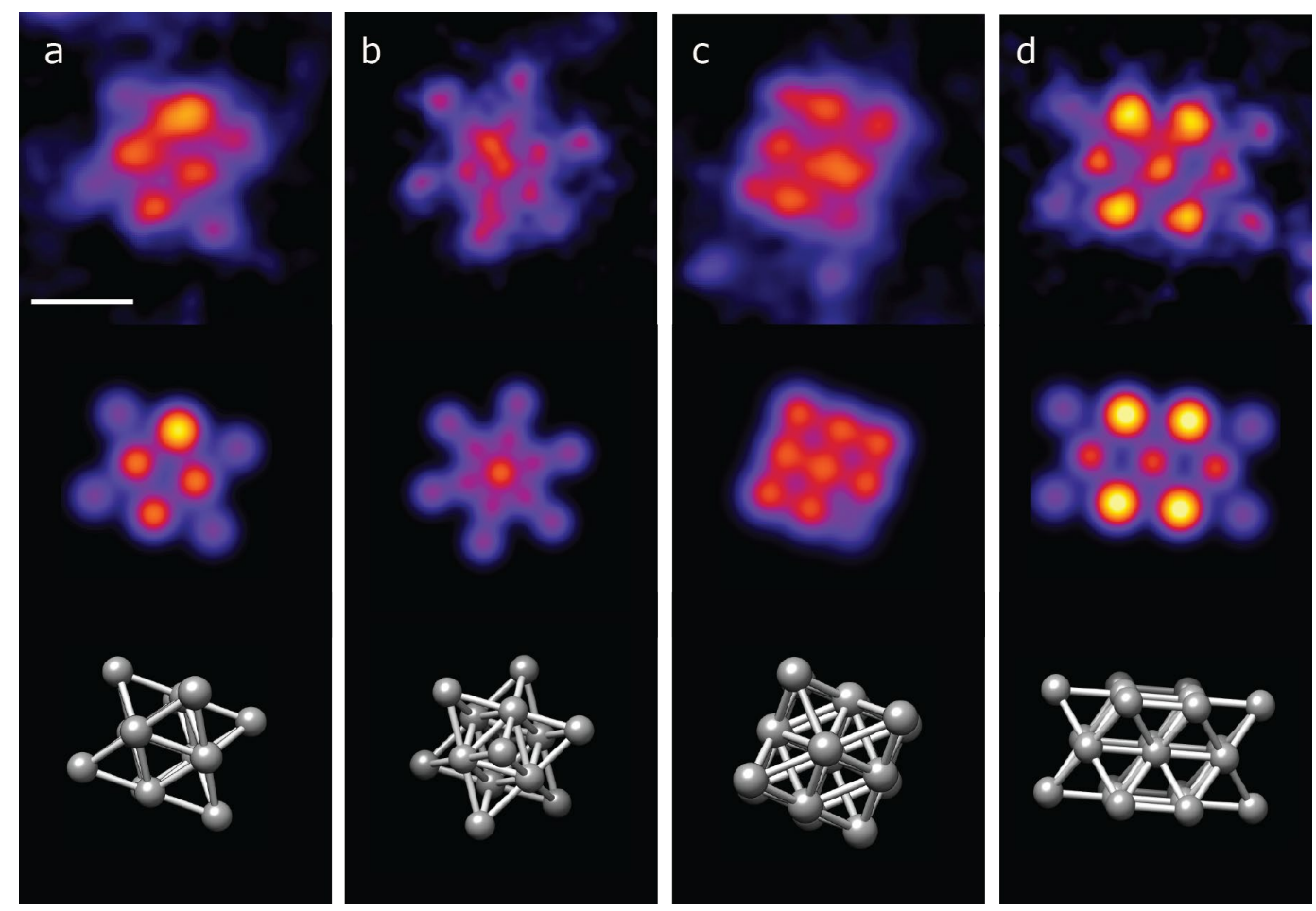

Figure 2: Representative cuboidal fcc clusters. Denoised images (top), simulation (middle) and models (below) of fcc cuboids. (a) A Pt ${ }_{13}$ cuboid (frame 1, Video S4). (b) A full $\mathrm{Pt}_{14}$ fcc unit cell with an additional mobile atom (frame 38, Video S5). (c) $\mathrm{A} \mathrm{Pt}_{17}$ rectangular cuboid (frame 22, Video S2). (d) A Pt 22 rectangular cuboid with two opposite edges truncated (frame 48, Video S6). $0.5 \mathrm{~nm}$ scale bar.

The observed fcc structures can be put in context to previous theoretical investigations, which indicate a co-presence of a multitude of different $\mathrm{Pt}_{n}$ structures whose properties are largely defined by their size and structural configuration, where the special case of $\mathrm{Pt}_{13}$ clusters has been discussed extensively. ${ }^{[20-26]}$ The magic-number stability of the closed-shell icosahedral structure has been predicted to give it a lower energy than close packed structures such as fcc and hexagonal close packed (hcp) configurations. ${ }^{[21,27]}$ We experimentally confirm for the first time structures that were recently theoretically predicted, ${ }^{[26,28]}$ namely the cuboid fcc, cuboctahedral fcc and hcp structures, different from the icosahedral geometry. In particular, the fcc cuboidal geometries are based on the 14-atom cube of the full fcc unit cell. In Fig. 3a-c, a $\mathrm{Pt}_{13}$ cuboid, missing one corner atom, is seen from three orientations. The fcc cuboctahedral structures are based on the 13-atom cuboctahedron, differing from the cuboid structures by having a more spherical shape, without the 3-coordinated corner atoms. Such a $\mathrm{Pt}_{13}$ cuboctahedral cluster, with one displaced atom, is seen in Fig. 3d. The hcp structured clusters are similar to the fcc cuboctahedrons, with the same 
coordination numbers of their atoms. However, the structure of hcp clusters are based on the 13-atom triangular orthobicupola, as the A-B-A stacking of hcp differs from the A-B-C stacking of fcc. An example of such an hcp cluster is seen in Fig. 3e, where the 15-atom cluster forms a triangular orthobicupola with two additional atoms attached. Surprisingly, a very clear and stable icosahedron has not been found. However, a good example of an icosahedral cluster is the $\mathrm{Pt}_{16}$ cluster in Fig. $3 \mathrm{f}$, with a 13 atom icosahedron-like core and three additional atoms attached.
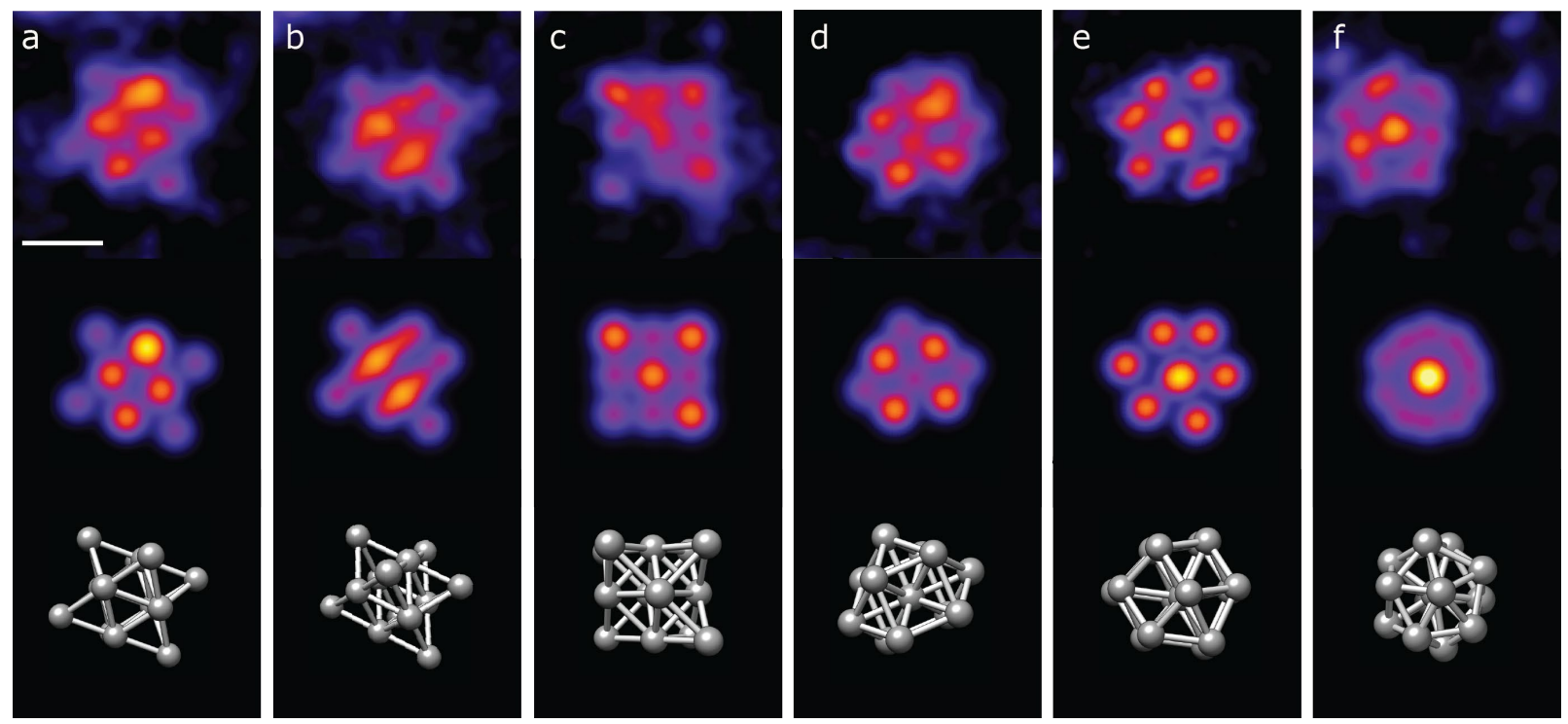

Figure 3: Four symmetries of Pt clusters. Denoised experimental STEM images (top), simulation (middle) and models (below) of the four different crystalline structures for Pt clusters at $350^{\circ} \mathrm{C}$. (a-c): Three orientations of a cuboidal $\mathrm{Pt}_{13}$ fcc cluster (frames 1,154 and 184, Video S4). (d): A Pt 13 fcc cuboctahedron with 1 atom displaced (frame 350, Video S7). (e) A Pt 15 hcp cluster (frame 122, Video S5). (f) An icosahedral-like $\mathrm{Pt}_{13}$ core surrounded by 5 additional atoms (frame 252, Video S8). $0.5 \mathrm{~nm}$ scale bar

To assess the stability of the four different geometries, namely the fcc cuboids and cuboctahedrals, hcp and icosahedrals, the 38 most stable clusters in the size range of $6-23$ atoms found in our experiments have been organized in Fig. 4 (see also Fig. S4). Each cluster was classified into one of the four categories if the structure could be formed by removing atoms from the original geometry (clusters smaller than 13 atom), or by adding atoms according to the same symmetry of the original (larger than 14 atoms). In this analysis, we assume the total integrated electron dose per area until the cluster changes or loses its structure, to be an indication of the stability of the cluster. The dose was estimated from the 
number of consecutive video frames showing each of the 38 clusters. Despite the fact that comparing cluster stability for different sizes is problematic, the reshaping of the data based on the observed crystalline structure (Fig. 4) allows capturing some trends. Notably, $66 \%$, i.e. 25 out of 38 clusters, were fcc structured, whereby $68 \%$ of these were fcc cuboids. When ordered by the four types of structures, (Fig. 4), there is an increasing stability icosahedral $\rightarrow \mathrm{hcp} \rightarrow \mathrm{fcc}$ cuboctahedral $\rightarrow$ fcc cuboid, with the most stable cluster being the 14-atom fcc cube. Due to the fragility of these clusters, we took care to minimize radiation exposure prior to recording, by briefly identifying clusters in the correct size range at a lower magnification (1-2 orders of magnitude lower dose rate). Therefore, clusters present in the first frame of a recording, are assumed to have formed naturally, while structures forming during a video are thought to have been significantly affected by radiation. Considering this, 11 of the 13 structures initially present were also cuboidal. Furthermore, when ordered by atomicity (Fig. S4), the most frequent and stable clusters were in the range of $12-15$ atoms, indicating an increased stability due to the magicnumber symmetry of the 13-atom icosahedron, triangular orthobicupola, cuboctahedron, and the 14-atom cube.

To further test these stability trends, the energetics of the four experimental geometries was investigated using DFT calculations at the PBE + D3 level. ${ }^{[26,28]} \mathrm{An}$ ab-initio treatment is needed, because of the limitations of the classical potentials in accurately treating such small systems (see SI). We carried out the computational study by comparing the potential energy trend of the clusters in gas phase, with the one of the clusters in interaction with a four-layered graphite substrate. The experimental structure of the surface of the amorphous carbon is unknown. For this reason, we approximated the local surface by a graphitic structure. This model is justified as amorphous carbon tends to become graphitic under electron irradiation. ${ }^{[29]}$ We focused on the energetics of the $\mathrm{Pt}_{13}$ clusters to compare the various structures deduced from the experimental data. In addition, the extraordinary stability of the $\mathrm{Pt}_{14}$ cube found in the experiments was verified via geometry relaxation both in vacuum and on a graphitic surface. A more detailed description of the simulated systems and on the computational details can be found in the Methods. 
As shown in Table 1, in gas phase all the geometries tested for $\mathrm{Pt}_{13}$ are stable, thus corresponding to local energy minima of the potential energy surface (PES). Among these, the cuboid is the lowest energy structure and the only one stable after chemisorption on the carbon surface. When optimized on the substrate, all the clusters but the fcc cuboid reach a mixed icosahedral/fcc structure (Fig. S5), which is lower in energy compared to the cuboid. This structure has not been observed experimentally and would be difficult to be distinguished from a disordered cluster due to the low symmetry. Noteworthy, the same geometry is also stable in gas phase, although higher in energy compared to the cuboid. This reverse trend is almost entirely due to the cluster adsorption energy, as shown in Table 1. DFT results give two important indications. The most stable experimental fcc structure (cuboid) reached by temperature annealing appears energetically stabilized on the carbon substrate. All other experimentally observed clusters are energetically unfavored, but may be entropically stabilized at high temperatures. Also important and not included in this analysis is the role of temperature in lowering the transition barriers from the "sea" of amorphous states to ordered crystalline structures, such as the ones observed in the experiments. Still, the fact that the cuboidal geometry is the most stable in terms of DFT potential energy, can be seen as a support to the experimental evidence that exactly this structure is the most persistent in the STEM observations.

Table 1: Potential energies of different cluster configurations. Potential energies (relative to the fcc cuboid) from $\mathrm{Pt}_{13}$ geometry optimization calculations at DFT/PBE+D3 of the four geometries experimentally found. The mixed icoshedral/fcc structure (see Fig. S5) is the final configuration for the cuboctahedron, the hcp and the icosahedron geometries after the geometry optimization on the substrate. For stable configurations on the graphitic substrate, the adsorption energy is also reported. All the energies are $\mathrm{eV}$.

\begin{tabular}{|l|l|l|l|}
\hline Geometry & Gas Phase & On Graphite & Adsorption Energy \\
\hline fcc, cuboid & 0. & 0. & 2.46 \\
\hline fcc, cuboctahedron & 2.76 & Not stable & Not stable \\
\hline $\begin{array}{l}\text { hcp, triangular } \\
\text { orthobicupola }\end{array}$ & 2.25 & Not stable & Not stable \\
\hline $\begin{array}{l}\text { icosahedron-like } \\
\text { mixed icosahedron/fcc }\end{array}$ & 2.80 & Not stable & Not stable \\
\hline
\end{tabular}




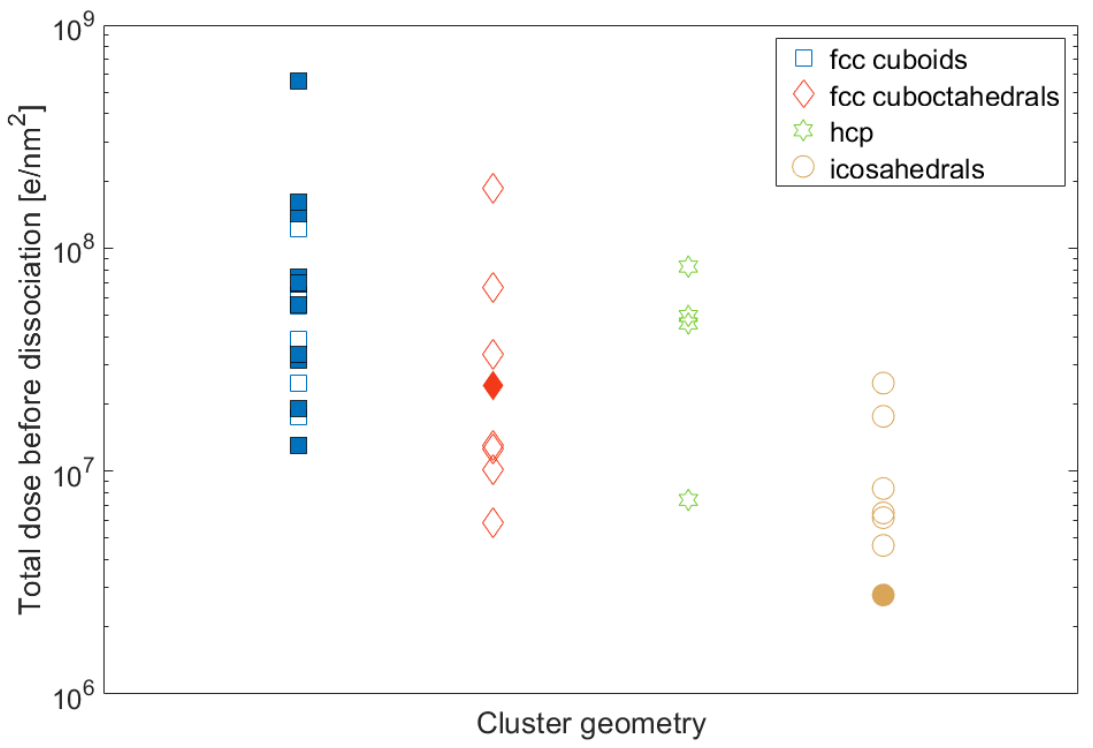

Figure 4: Stability of different cluster structures and geometries. Experimentally observed stability (total dose before transformation) of the 38 most stable clusters of different atomicities and structures identified in this work. Filled dots are clusters that were present in the initial frame of the video, and thus assumed to have formed naturally, while the hollow dots are clusters that formed during irradiation. The 38 clusters ordered by the four types of geometries, showing a decreasing stability fcc cuboidal $\rightarrow$ fcc cuboctahedral $\rightarrow$ hcp $\rightarrow$ icosahedral. 


\section{CONCLUSIONS}

Based on the results and analyses above, we can conclude the following:

- We demonstrated a fast scanning approach in atomic-resolution electron microscopy to study the atomic structure of carbon-supported sub-nm $\mathrm{Pt}_{\mathrm{n}}$ clusters. This was made possible by using the spatio-temporal denoising of PGURE-SVT ${ }^{[18]}$.

- For the first time, we demonstrated a proof-of-concept method to assess the stability of the crystalline clusters at elevated temperatures, which showed that the clusters have a strong preference towards fcc geometries down to six atoms in size. The most stable clusters exhibited cuboidal structures, whose stability was further confirmed by DFT simulations.

- We experimentally showed the existence of the smallest possible seeds for nanoparticles of the cubic, cuboctahedral and octahedral geometries.

- We investigated Pt (and $\mathrm{Au}$ ) on $\mathrm{C}$, showing that an amorphous-to-crystalline transition occurs at elevated temperatures, which is not the case with Pt on SiN. Electron beam, temperature and substrate seem to be critical factors, but clearly more work (alternative experimental techniques) is needed to fully understand this phenomenon and its possible fundamental implications.

Overall, our results reveal new structural characteristics of sub-nm Pt clusters, which are particularly important to further understand the catalytic behaviour of Pt on C. 


\section{References}

[1] G. Schmid, Nanoparticles: From Theory to Application, Second Edition, Wiley-VCH Verlag GmbH \& Co. KGaA, Weinheim, Germany, 2010.

[2] S. A. Claridge, A. W. Castleman, S. N. Khanna, C. B. Murray, A. Sen, P. S. Weiss, ACS Nano 2009, 3, 244.

[3] S. Vajda, M. J. Pellin, J. P. Greeley, C. L. Marshall, L. A. Curtiss, G. A. Ballentine, J. W. Elam, S. CatillonMucherie, P. C. Redfern, F. Mehmood, P. Zapol, Nat. Mater. 2009, 8, 213.

[4] J. Wei, E. Iglesia, J. Phys. Chem. B 2004, 108, 4094.

[5] T. Imaoka, Y. Akanuma, N. Haruta, S. Tsuchiya, K. Ishihara, T. Okayasu, W.-J. Chun, M. Takahashi, K. Yamamoto, Nat. Commun. 2017, 8, 688.

[6] R. Siburian, T. Kondo, J. Nakamura, J. Phys. Chem. C 2013, 117, 3635.

[7] N. Cheng, S. Stambula, D. Wang, M. N. Banis, J. Liu, A. Riese, B. Xiao, R. Li, T.-K. Sham, L.-M. Liu, G. A. Botton, X. Sun, Nat. Commun. 2016, 7, 13638.

[8] C. Lemire, R. Meyer, S. Shaikhutdinov, H.-J. Freund, Angew. Chemie Int. Ed. 2004, 43, 118.

[9] L. D. Marks, Reports Prog. Phys. 1994, 57, 603.

[10] A. S. Barnard, N. P. Young, A. I. Kirkland, M. A. van Huis, H. Xu, ACS Nano 2009, 3, 1431.

[11] K. Koga, K. Sugawara, Surf. Sci. 2003, 529, 23.

[12] H. Zheng, R. K. Smith, Y. Jun, C. Kisielowski, U. Dahmen, A. P. Alivisatos, Science (80-. ). 2009, 324, 1309.

[13] P. D. Nellist, S. J. Pennycook, Science (80-. ). 1996, 274, 413.

[14] Z. Y. Li, N. P. Young, M. Di Vece, S. Palomba, R. E. Palmer, a L. Bleloch, B. C. Curley, R. L. Johnston, J. Jiang, J. Yuan, Nature 2008, 451, 46.

[15] S. Van Aert, K. J. Batenburg, M. D. Rossell, R. Erni, G. Van Tendeloo, Nature 2011, 470, 374.

[16] S. Bals, S. Van Aert, C. P. Romero, K. Lauwaet, M. J. Van Bael, B. Schoeters, B. Partoens, E. Yücelen, P. Lievens, G. Van Tendeloo, Nat. Commun. 2012, 3, 897.

[17] J. Lee, W. Zhou, S. J. Pennycook, J.-C. Idrobo, S. T. Pantelides, Nat. Commun. 2013, 4, 1650.

[18] T. Furnival, R. K. Leary, P. A. Midgley, Ultramicroscopy 2017, 178, 112.

[19] T. Furnival, R. K. Leary, E. C. Tyo, S. Vajda, Q. M. Ramasse, J. M. Thomas, P. D. Bristowe, P. A. Midgley, Chem. Phys. Lett. 2017, 683, 370.

[20] J. P. K. Doye, D. J. Wales, New J. Chem. 1998, 22, 733.

[21] T. Imaoka, H. Kitazawa, W.-J. Chun, S. Omura, K. Albrecht, K. Yamamoto, J. Am. Chem. Soc. 2013, 135, 13089.

[22] C. L. Heredia, V. Ferraresi-Curotto, M. B. López, Comput. Mater. Sci. 2012, 53, 18. 
[23] I. Fampiou, A. Ramasubramaniam, J. Phys. Chem. C 2012, 116, 6543.

[24] S. H. Yang, D. A. Drabold, J. B. Adams, P. Ordejón, K. Glassford, J. Phys. Condens. Matter 1997, 9, L39.

[25] V. Kumar, Y. Kawazoe, Phys. Rev. B 2008, 77, 205418.

[26] V. Fung, D. Jiang, J. Phys. Chem. C 2017, 121, 10796.

[27] D. Tománek, S. Mukherjee, K. H. Bennemann, Phys. Rev. B 1983, 28, 665.

[28] A. Nie, J. Wu, C. Zhou, S. Yao, C. Luo, R. C. Forrey, H. Cheng, Int. J. Quantum Chem. 2007, $107,219$.

[29] T. Aizawa, E. Iwamura, MRS Proc. 2006, 960, 0960.

[30] R. Erni, H. Heinrich, G. Kostorz, Ultramicroscopy 2003, 94, 125.

[31] T. R. Henninen, "peak-of-histogram," can be found under https://github.com/hentr/peak-of-histogram, 2019.

[32] S. Van Aert, A. De Backer, G. T. Martinez, B. Goris, S. Bals, G. Van Tendeloo, A. Rosenauer, Phys. Rev. B 2013, 87, 064107.

[33] A. De Backer, K. H. W. van den Bos, W. Van den Broek, J. Sijbers, S. Van Aert, Ultramicroscopy 2016, 171, 104.

[34] F. Wang, A. Eljarrat, J. Müller, T. Henninen, E. Rolf, C. Koch, arXiv Prepr. 2019.

[35] F. Wang, T. R. Henninen, "STEM image denoising by artificial neural network," can be found under https://github.com/fengwang/nn-denoising, 2019.

[36] J. Hutter, M. Iannuzzi, F. Schiffmann, J. VandeVondele, Wiley Interdiscip. Rev. Comput. Mol. Sci. 2014, 4, 15.

[37] J. VandeVondele, J. Hutter, J. Chem. Phys. 2007, 127, 114105.

[38] M. Krack, Theor. Chem. Acc. 2005, 114, 145.

[39] J. P. Perdew, K. Burke, M. Ernzerhof, Phys. Rev. Lett. 1996, 77, 3865.

[40] S. Grimme, J. Antony, S. Ehrlich, H. Krieg, J. Chem. Phys. 2010, 132, 154104.

[41] J. J. Panek, A. Jezierska-Mazzarello, 2013, pp. 287-297. 


\section{METHODS SECTION}

\section{Experimental details}

Samples were made by plasma sputtering Pt onto Protochips Fusion thermal chips (Leica ACE200: 10-2 mbar $\mathrm{N}_{2}, 15 \mathrm{~mA}$ plasma current, $1 \mathrm{~s}$ shutter time, ca $12 \mathrm{~cm}$ working distance), which reproducibly produces a low-density scatter of individual atoms and small clusters up to ca $1.5 \mathrm{~nm}$ in diameter, on the 10-20 nm thick amorphous carbon film on the chip. STEM was done using a probe corrected FEI Titan Themis at $300 \mathrm{kV}$. Heating experiments were performed using a Protochips Fusion heating holder, from room temperature up to $600^{\circ} \mathrm{C}$. To image the clusters at room temperature, carbon contamination was cleared by flash heating to $500^{\circ} \mathrm{C}$ for $1 \mathrm{~s}, 1-5$ times depending on amount of contamination. This was not observed to affect the structure of the clusters.

Time series were recorded with pixel sizes of $6.3-12.5 \mathrm{pm}$, dwell times of 50-150 ns, and frame sizes of $512 \times 512$ pixels (14-20 fps), 256x256 (35-43 fps), or 128x128 (74-147 fps). The recordings were of 5004000 frames, corresponding to ca $30-60$ seconds of recording time at the various frame rates and electron beam currents of 40-90 pA. At these fast scan rates, the microscope software (TIA, Velox) overestimated the imaging frame rate. Therefore, the actual frame rate was measured by counting the actual number of frames recorded in one minute for the various scanning parameters. Temperatures and frame rate of the various Videos S1-S9 are noted in Tab. S1.

To image at a lowest beam dose, 15 pA probe current, $600 \mathrm{~ns}$ dwell time, $512 \times 512$ pixels, and $8.8 \mathrm{pm}$ pixel size, was used, enabling recording at $2.5 \mathrm{fps}$ with a beam dose of $\sim 5 \times 10^{5} \mathrm{e} / \mathrm{nm}^{2} \mathrm{~s}$. This is compared to $\sim 6 \times 10^{6} \mathrm{e} / \mathrm{nm}^{2} \mathrm{~s}$ for the typical microscope parameters used in this work, with the highest dose of $\sim 5 \times 10^{7} \mathrm{e} / \mathrm{nm}^{2} \mathrm{~s}$ for recordings at $147 \mathrm{fps}$. Attempts to image clusters at $80 \mathrm{kV}$ acceleration voltage gave insufficient resolution to observe the dynamics of the individual atoms in the clusters, and were therefore difficult to compare to the $300 \mathrm{kV}$ recordings.

The beam dose rate was observed to affect the crystallinity, as little crystallinity was seen at a high dose of $5 \times 10^{7} \mathrm{e} / \mathrm{nm}^{2} \mathrm{~s}$ (Video S3), compared to the typical dose in these experiments of $\sim 6 \times 10^{6} \mathrm{e} / \mathrm{nm}^{2} \mathrm{~s}$. When 
imaging at a lower dose of $\sim 5 \times 10^{5} \mathrm{e} / \mathrm{nm}^{2} \mathrm{~s}$, a few cases of crystalline clusters were also found at $200^{\circ} \mathrm{C}$ (Video S9), but at such a low dose acquiring interpretable data was difficult, even when considering the denoising algorithm. Yet, recording at a high frame rate is necessary, as recording at the low frame rate of $2 \mathrm{fps}$, was not sufficient to record multiple frames at one orientation for more reliably interpreting the cluster structure.

\section{STEM image simulations}

Simulations of STEM images for image interpretation were done with a custom written frozen-phonon multislice code. ${ }^{[30]}$

\section{Image Processing}

To enable the structural analysis of the noisy raw data, the experimental videos were denoised using PGURE-SVT, applying spatio-temporal correlation.[18] This was followed by Gaussian filter, with $\sigma$ depending on the videos' pixel size ( $\sigma=2.8,4$ and 5 , for respectively pixel sizes $=12.5,8.8$ and $6.2 \mathrm{pm})$, and a peak-of-histogram background subtraction ${ }^{[31]}$. The images in Figure 1-3 were false-coloured by a modified 'Fire' lookup table in Fiji/lmageJ, where the contrast was normalized so that the colours approximately correspond to the number of atoms overlapping: 1 atom - blue/purple, 2 atoms red/orange, 3 atoms - yellow.

\section{D interpretation}

Interpretation of the 3D structures of the Pt clusters, and thus extracting crystallographic information, requires a special approach. As nearly all recorded frames are dominated by atoms partially overlapping each other, using known quantification approaches ${ }^{[2,33]}$ failed to extract reliable 3D data of the clusters. Since we analyze a single atomic species $(\mathrm{Pt})$ with individual atoms always present on the support film, the STEM contrast could still be quantified to distinguish between "columns" of 1-5 overlapping atoms. However, due to the low SNR of the original recordings, the contrast is not fully consistent from frame to frame of the denoised videos. Therefore, the image contrast can only be understood correctly by studying multiple consecutive frames with relatively little cluster dynamics, or by blending multiple consecutive 
frames. With this strategy, we successfully developed 3D structural models of selected Pt clusters and performed multi-slice simulations of STEM images, whose comparisons with our experimental data qualitatively validated the structure models. Also notably, all the clusters were also interpreted identically when the data was denoised using an in-house neural network-based denoiser. ${ }^{[34,35]}$

For the sake of clarity, we give an example of how to directly interpret the structure of the easily interpretable cluster in Fig. 3a-c: 1) Find the exact number of atoms in a cluster by interpreting the contrast and counting atoms through tens of frames (which can be assisted by StatSTEM ${ }^{[32,33]}$ ) and accounting for any atoms attaching onto or detaching from the cluster. The exact number of atoms is found if the vast majority of frames gives the same number. This example cluster had 13 atoms (Fig. 3a-c upper row). 2) Knowing the number of atoms in the cluster, hypothetical atomic models can be built (Fig. 3a-c bottom row). 3) For the most stable clusters, the model can be compared at multiple orientations, such as the three orientations seen for this example. 4) Verify the interpreted model by comparing select frames with simulated images (Fig. 3a-c middle row). It must be emphasized that Figs. 1-3 show comparisons of single STEM images with simulated images of models, which were not found by analyzing a single image, but by analyzing series of tens to hundreds of images.

To generate the plots of the 38 most stable clusters in Figs. 4 and S4, in total ca 3500 frames were manually interpreted. Some limitations of this quantitative analysis should be mentioned. The data was limited in upper size range, as both the number of hypothetical cluster structures and the difficulty of interpreting the image contrast, increases with the number of atoms in a cluster. The number of data points was limited by the large amount of time required for the manual analysis, but no automated algorithm capable of this interpretation exists, as this is a non-trivial problem. Furthermore, this analysis has an inherent bias in that structures with highly symmetric projections at many orientations (primarily fcc structures), are easier to identify and interpret than less symmetric structures (hcp, icosahedrals and most other hypothetical structures). It is therefore possible that some structured clusters were missed, but also that frames showing icosahedral-like and hcp-like structures were misinterpreted as hcp and icosahedral structures, and thus their stability might have been over- rather than underestimated. Nevertheless, it is 
unlikely that any clusters with a stability above $5 \times 10^{7} \mathrm{e} / \mathrm{nm}^{2} \mathrm{~s}$ were missed in the analyzed data. Additional details regarding the 3D structure interpretation are given in the Supplementary Materials S.4.

\section{Ab-initio simulations}

Simulation Protocol. At first, the geometries of the four $\mathrm{Pt}_{13}$ clusters presented in Table 1 were optimized in gas phase and subsequently on an $8 \times 8 \times 2$ slab of graphite with a $10 \AA$ vacuum. The corresponding cell simultaneously assures the convergence of the sampling in k-space (SI: Section S.6), and avoids the interaction between the periodic images of Pt clusters. The center of mass of each cluster was initially positioned on the three different adsorption sites, i.e. top, bridge, and hollow, and the total geometry of the system was optimized, keeping the two bottom layers of graphite fixed (bulk-optimized conditions). For each $\mathrm{Pt}_{13}$ configuration and possible adsorption position, we used three different initial rotations of the $\mathrm{Pt}_{13}$. This initial (although limited) conformational search allows a better identification of the lowest energy configuration (reported in Table 1) in every PES minimum, thus providing a better energy comparison between all the possible geometries. A similar simulation protocol was adopted for the $\mathrm{Pt}_{14}$ cuboid cluster.

Electronic Structure Details. Density functional theory (DFT) simulations were conducted using the CP2K package. ${ }^{[36]}$ Valence electrons were treated explicitly using the DZVP basis set, ${ }^{[37]}$ whereas interactions with frozen atom cores were described with the GTH pseudopotentials. ${ }^{[38]}$ The charge-density cutoff for the auxiliary plane-wave basis was set to $600 \mathrm{Ry}$, and the $\mathrm{PBE}^{[39]}$ was adopted as exchangecorrelation functional. The Grimme empirical correction scheme (DFT-D3) $)^{[40]}$ was used to include the dispersion interactions (vdW attraction) essential in graphite, ${ }^{[41]}$ and in the description of adsorption processes. To be consistent in the comparison, these were also adopted in the simulation of the freestanding Pt clusters (in vacuum). 


\section{Acknowledgments}

We acknowledge funding from the European Research Council (ERC) under EU's Horizon 2020 program (grant agreement No. 681312).

\section{Author contributions}

T.R.H and R.E conceived the research work. T.R.H conducted the experiments and analyzed the data.

F.W did the denoising. M.B and D.P carried out the DFT and MD calculations. The manuscript was written by T.R.H and R.E and all authors contributed to it.

\section{Data availability}

All pertinent data presented (raw and processed) are available from the corresponding authors upon reasonable request or can be downloaded from Zenodo at https://zenodo.org/record/3360503 (DOI: 10.5281/zenodo.3360503) 